\title{
Diseño organizacional de la escuela de policia - San Bartolo - Perú
}

\section{Organizational design of the police school - San Bartolo - Peru}

Emigdio Melquiades Zavaleta Cabrera ${ }^{1}$ 0000-0002-2359-0483

${ }^{1}$ Universidad Nacional del Callao, Perú.

ezavaleta1804@gmail.com

Recibido el: 25/09/2021

Aceptado el: 10/11/2021

Publicado el: 15/12/2021

\section{Resumen}

El propósito de la investigación es determinar las diferentes percepciones en el nivel del diseño organizacional, por secciones, en la Escuela de Policía San Bartolo (EPSB), en una muestra de 120 alumnas. Se presenta un estudio de enfoque cuantitativo, no experimental, transeccional y descriptivo. Los resultados permiten identificar los niveles de diseño organizacional en sus diferentes dimensiones, con un Alfa de Cronbach de confiabilidad de 0.91, aplicación del estadístico de prueba Kruskal Wallis para evaluar la hipótesis; observándose que el $95.9 \%$ de las encuestadas perciben un nivel moderado y alto en el diseño organizacional; el $89,1 \%, 97,5 \%$ y $93,3 \%$, respectivamente un nivel moderado y alto en las dimensiones formalización, profesionalismo 22 cultura; y, el $58,3 \%, 32,5 \%$, y $24,2 \%$, respectivamente, un nivel bajo en las dimensiones tecnología, entorno, metas y estrategias. En conclusión, se evidencian que existen diferentes percepciones al nivel del diseño organizacional, con un nivel moderado y alto en la formalización, profesionalismo y cultura y un nivel bajo en la tecnología, metas y estrategia. Por ello, es relevante cubrir estas falencias con la necesidad de plataforma tecnológica, estrategia importante y prioritaria en la gestión que permite mejorar la capacidad de respuesta organizacional y el cumplimiento de metas y objetivos.

Palabras clave: Diseño organizacional; toma de decisiones; estructura organizacional; estrategia; objetivos.

\begin{abstract}
The purpose of the research is to determine the different perceptions at the level of organizational design, by sections, in the San Bartolo Police School (EPSB), in a sample of 120 female students. A study with a quantitative, non-experimental, transectional and descriptive approach is presented. The results allow identifying the levels of organizational design in its different dimensions, with a reliability Cronbach's Alpha of 0.91, application of the Kruskal Wallis test statistic to evaluate the hypothesis; observing that $95.9 \%$ of those surveyed perceive a moderate and high level in the organizational design; $89.1 \%$, $97.5 \%$ and $93.3 \%$, respectively, a moderate and high level in the formalization, professionalism and culture dimensions; and $58.3 \%, 32.5 \%$, and $24.2 \%$, respectively, a low level in the technology, environment, goals and strategies dimensions. In conclusion, it is evident that there are different perceptions at the level of organizational design, with a moderate and high level in formalization, professionalism and culture and a low level in technology, goals and strategy. Therefore, it is important to cover these shortcomings with the need for a technological platform, an important and priority strategy in management that allows improving the organizational response capacity and the fulfillment of goals and objectives.
\end{abstract}

Keywords: Organizational design; decision making; organizational structure; strategy; goals. 


\section{Introducción}

Las reformas administrativas están orientadas a mejorar las intervenciones del estado para generar bienestar en la sociedad (Ugarte et al., 2021). La Policía Nacional del Perú (PNP), siempre ha estado sujeta por diferentes Gobiernos de turno a constantes reorganizaciones y reestructuraciones para hacer más eficiente la labor policial, medidas que se extienden hasta el primer nivel del sistema educativo policial, siendo una de ellas la Escuela de Policía San Bartolo (EPSB), en donde se forman las futuras policías de la PNP. Cuando las reorganizaciones y reestructuraciones son frecuentes en una organización, es porque su estructura a través de la cual están dispuestos sus diferentes órganos y que es definida por su diseño organizacional no es adecuada a sus necesidades (Chiavenato, 2006). Por ello, es importante contar con un diseño organizacional adecuado que permita la aplicación de metas y estrategias operacionales. Todas las instituciones deben tener un orden jerárquico en donde se establece la cadena de mando y toma de decisiones, que orienten el trabajo que se realiza (Cornejo y Flores, 2020).

Actualmente existen organizaciones que vienen realizando cambios a través del análisis de su estructura y adaptarse a procesos inherentes a sus actividades (Pacheco, et al., 2020). En el marco del planeamiento estratégico, que define el diseño organizacional, países como Chile, Brasil, Colombia, entre otros, nos llevan 25 años en implementación de la calidad de los sistemas. Nuestro país inicia en el año 1962, la planificación de los grandes objetivos nacionales, ejecutando metas y objetivos totalmente desarticulados en todos los niveles de gobierno, donde la PNP no es ajena a esta situación; teniendo en consideración que, en todo el mundo, la institución policial es fundamental para la aplicación de los esfuerzos de neoliberalización (Buderath y Heath, 2021). A pesar de ello, no se le ha dado la debida importancia, existiendo escaso interés de estas instituciones por promover desde su interior estudios desde las ciencias sociales (Barrera, 2016).

Nuestro país atraviesa una etapa de consolidación democrática que exige modernizar y reformar las instituciones del Estado. La PNP ha merecido una atención especial ante el incremento de situaciones que amenazan el orden interno, así como por la aparición de nuevas formas delictivas y proliferación de conflictos sociales que demandan nuevas estrategias desde el nivel de formación policial y por lo tanto nuevos diseños organizacionales para enfrentar con éxito dicha situación. El avance de la ciencia y la tecnología crean en las organizaciones cambios sustanciales en las estrategias y actividades de desarrollo (Besri y Boulmakoul, 2021). Este desarrollo, genera que los conocimientos queden obsoletos con mayor rapidez (Palomeque y Ruiz, 2019). Siendo primordial que la institución policial esté preparada para afrontar cambios constantes y poder superar las ineludibles dificultades culturales, estructurales y políticos (Bond, 2020).

En ese sentido, se han aprobado lineamientos que definen el diseño, estructura, organización $\mathrm{y}$ funcionamiento de las entidades del Estado; entre ellas la PNP, que satisfaga adecuada y oportunamente las necesidades de orden interno, seguridad ciudadana y protección de las personas y comunidad. El sistema educativo de la PNP, en la que está inmerso la EPSB, debe diseñar las metas, las estrategias educativas y el diseño organizacional que permita alcanzar los objetivos organizacionales y mejorar la calidad de la formación de los alumnos de las escuelas de policías. Los colaboradores juegan un rol importante, se deben desarrollar sus habilidades que coadyuven a buenos resultados en la gestión (Diaz et al., 2018). Este desarrollo debe ser gestionado adecuadamente, en razón de tener una repercusión positiva en la seguridad social de un país (Lorek et al. 2020); y, que se deben aplicar las estrategias, manejo eficiente de los recursos y el talento humano, como factor importante en el logro de las metas organizacionales (Serkan et al., 2019).

Se requiere de condiciones adecuadas para el desarrollo de los procesos de formación, como una preocupación constante para generar el cambio en las organizaciones policiales (Willis et al., 2020). Revertir la percepción de inseguridad ciudadana como un problema medular de las sociedades de la posmodernidad, donde se observa aumento de la violencia criminal, con una tasa de ocho homicidios por cada 100,000 habitantes a nivel mundial y 24,5 homicidios por cada 100,00 habitantes en América Latina, que conforme la opinión de 448 lideres y periodistas de catorce países de Latinoamérica y El Caribe, es uno de los problemas principales que asediaron la región (Quintero, 2020). En el Perú, la percepción de inseguridad ciudadana supera el $86 \%$ y que exige efectividad en el desempeño de las funciones (Estupiñan et al., 2018). La creciente 
ola delictiva por parte de grupos criminales que ha causado zozobra en la población, requiere mayor atención de la PNP que demanda de una estrategia y efectividad de sus efectivos policiales. En ese sentido, la siguiente investigación buscó determinar las diferentes percepciones en el nivel del diseño organizacional en la EPSB, para entender cómo funciona la organización y si el diseño organizacional obedece a las estrategias que se vienen aplicando.

\section{Marco teórico}

Existe una relación significativa entre el diseño y el desarrollo organizacional (Barona y Ramírez, 2020) y que este contribuye en la mejora de la gestión (Castro, 2020). En ese sentido, se debe definir la forma más eficaz de la organización para ejecutar las estrategias más adecuadas para el logro de metas y objetivos organizacionales; siendo fundamental desarrollar estrategias y herramientas en la gestión para el mejor desempeño de sus colaboradores y lograr una posición competitiva de la organización en un mundo globalizado que avanza vertiginosamente (Vera y Blanco, 2019). El diseño organizacional "es un proceso en el cual los gerentes toman decisiones para elegir la estructura organizacional adecuada para la estrategia de la organización y el entorno en el cual los miembros de la organización ponen en práctica dicha estrategia" (Stoner et al., 1999, p. 355).

Gareth (2008) también define el concepto de diseño organizacional como el proceso mediante el cual se toman decisiones para definir los aspectos relacionados con la estructura y la cultura, y son responsabilidad de los gerentes en una organización, que posibilitan el control de todas las actividades en sus diferentes niveles para el cumplimiento de sus metas y objetivos organizacionales. Son aspectos que definen el diseño organizacional, la disposición de los órganos en los diferentes niveles de la estructura organizacional, que actúan coordinadamente y mantienen una relación de interdependencia para el desarrollo de sus diferentes actividades, con las cuales la organización pueda lograr sus objetivos trazados (Chiavenato, 2006). Por lo tanto, conforme mencionaron Dammert y Castañeda (2019), se deben generar estructuras organizacionales que respondan a la implementación de la estrategia.

Losenfoquesconceptualesdeldiseñoorganizacional expuestas en los párrafos precedentes, coinciden en definir este concepto como el proceso para la toma de decisiones respecto a la elección, la búsqueda y la configuración de la estructura organizacional adecuada para el desarrollo de las actividades hacia el logro de los propósitos de la organización. En las organizaciones, las características específicas del diseño organizacional, que para nuestro caso materia de investigación, es la variable de estudio, están definidas por sus dimensiones, conforme lo mencionó Daft (2010) cuando señaló: "estas dimensiones describen a las organizaciones de manera similar a como los rasgos de personalidad y físicos describen a las personas" (p. 14). Se ha identificado conforme lo definió Daft (2010), que "las dimensiones del diseño organizacional son: la formalización, el profesionalismo, la tecnología, el entorno o medio ambiente, las metas y estrategias, y la cultura" (p. 15).

En ese orden de ideas, Daft (2010) también hace mención a las dimensiones y las conceptualiza en "la formalización se refiere a la cantidad de documentación escrita en la organización" (p. 15); el profesionalismo como "el nivel de educación formal y capacitación de los empleados"; la tecnología "se refiere a las herramientas, técnicas y acciones empleadas para transformar los insumos en productos"; el entorno "incluye todos los elementos fuera de la organización"; "las metas y las estrategias de una organización definen el propósito y las técnicas competitivas que la diferencian de otras organizaciones"; $y$, la cultura como "el conjunto subyacente de valores, creencias, entendimientos y normas clave compartidos por los empleados" (p. 17).

Partiendo del concepto que las escuelas de formación de la Policía Nacional del Perú, son los órganos de ejecución y gestión académica, encargados de planear, dirigir, organizar, coordinar, controlar, evaluar, y conducir la etapa de formación de los alumnos, futuros policías; resulta de imperiosa necesidad que la escuela de la PNP materia de investigación, para desarrollar sus actividades a través de este proceso administrativo, cuente con una estructura organizacional que permita la ejecución de su plan estratégico que conduzca a resultados de efectividad en la gestión.

\section{Metodología}

Se presenta una investigación con enfoque cuantitativo, conforme lo definiera Hernández et al. (2014) "utiliza la recolección de datos para 
probar hipótesis con base en la medición numérica y el análisis estadístico, con el fin de establecer pautas y probar teorías" (p. 4); con un diseño no experimental, transeccional y descriptivo, porque se realiza sin manipular la variable "diseño organizacional" y observando los fenómenos tal como se presentan en su contexto natural, y porque la recolección de datos se realiza en un solo momento, analizando los problemas tal y cómo se encuentran al momento de la investigación (Hernández et al., 2014).

Participantes. La muestra del estudio fue de 120 alumnas provenientes de una población de 171 alumnas, compuesta en tres secciones de estudio (A, B y C), cuyas edades oscilaron entre 19 y 21 años, distribuida conforme la tabla 1. Se utilizó la técnica del muestreo probabilístico, conforme lo definieron Hernández et al. (2014) es un subgrupo de la población en la que todos sus elementos tienen la misma probabilidad de ser elegidos y son esenciales en los diseños de investigación transeccionales.

Tabla 1. Distribución de la muestra.

\begin{tabular}{lcc}
\hline Secciones & Alumnas/sección & Muestra \\
\hline Sección A & $54 \times 0.70$ & 38 \\
\hline Sección B & $60 \times 0.70$ & 42 \\
\hline Sección C & $57 \times 0.70$ & 40 \\
\hline Total & 171 & 120 \\
\hline
\end{tabular}

Técnica de recolección de datos. Encuesta a las alumnas de la EPSB con el propósito de conocer las percepciones en relación al objeto de estudio. Se utilizó como instrumento el cuestionario, con 30 ítems, empleando la escala de medición de actitudes tipo Likert, en cinco categorías, del 1 (Muy en desacuerdo), al 5 (Muy de acuerdo), que "consiste en un conjunto de ítems presentados en forma de afirmaciones o juicios, ante los cuales se pide la reacción de los participantes" (Hernández et al., 2014, p. 238). La validez del instrumento fue sometido a juicio de tres expertos. El resultado de la validez del instrumento fue aplicable. El instrumento utilizado fue sometido a la prueba de confiabilidad de Alpha de Cronbach: $\infty=\left[\frac{K}{K-1}\right]\left[1-\frac{\sum_{i=1}^{k} S_{i}^{2}}{S_{t}^{2}}\right]$, donde $k$ es el número de ítems (preguntas) del instrumento (cuestionario), $s_{i}^{2}$ : es la varianza de los ítems (desde $1 \ldots$ i) y $s_{t}^{2}$

es la varianza total del cuestionario (suma de las varianzas de los evaluadores); el análisis de consistencia varía entre 0 y 1 . Según interpretación de Valderrama (2013), si el valor de es de 0.60 hacia arriba se aproxima a ser confiable, y si es de 0.80 hacia arriba es altamente confiable. En la prueba, el índice resultó 0.91 , por ende, el instrumento utilizado es altamente confiable.

Procedimiento de análisis de datos. Teniendo en consideración que el estudio se realizó en el contexto policial, donde la información tiene el carácter reservado y confidencial, las participantes fueron alumnas de la EPSB, quienes accedieron en forma voluntaria y anónima, llevando a cabo un procedimiento de consentimiento informado, dando a conocer los objetivos y características del estudio, desarrollando el cuestionario directamente en el aula, dando su consentimiento explícito de participación. Para la realización de los análisis estadísticos pertinentes se empleó el paquete estadístico SPSS. Primero, se realizó el análisis de los niveles porcentuales de la variable diseño organizacional, por secciones, para hacer una comparación del diseño organizacional de la EPSB. Posteriormente, se realizó el análisis de los niveles porcentuales de las dimensiones del diseño organizacional y hacer la comparación porcentual correspondiente.

Para evaluar la hipótesis, se aplicó el estadístico de prueba de Kruskal Wallis, conforme lo definió Quispe etal.,(2019) es un estadístico no paramétrico para probar si un grupo de datos proviene de la misma población y que se utiliza para varias muestras independientes, para comparar más de dos grupos de rangos (medianas) y determinar que la diferencia no se deba al azar (que la diferencia sea estadísticamente significativa).

\section{Resultados}

Mediante las encuestas aplicadas a las alumnas de la EPSB, Secciones A, B y C, se obtuvieron los resultados señalados en la tabla 2 . Se aprecian los niveles porcentuales de la variable diseño organizacional por secciones, en donde el $95.9 \%$ 
de las encuestadas perciben un nivel moderado $\mathrm{y}$ alto en el diseño organizacional, lo cual es relativamente favorable por las decisiones que realiza el director para el logro de los objetivos organizacionales. En la tabla 3, se presentan los niveles porcentuales del diseño organizacional por dimensiones como son la formalización, profesionalismo, tecnología, entorno o medio ambiente, metas y estrategias, y cultura.

Tabla 2. Niveles porcentuales del diseño organizacional por secciones.

\begin{tabular}{|c|c|c|c|c|c|}
\hline \multirow[b]{2}{*}{ Variable } & \multirow[b]{2}{*}{ Niveles } & \multicolumn{3}{|c|}{ Secciones } & \multirow[b]{2}{*}{$\begin{array}{c}\text { Total } \\
\% \\
\text { Secciones }\end{array}$} \\
\hline & & $\begin{array}{c}\text { A } \\
\% \text { Dentro } \\
\text { de Sección }\end{array}$ & $\begin{array}{c}\text { B } \\
\% \text { Dentro } \\
\text { de sección }\end{array}$ & $\begin{array}{c}\text { C } \\
\% \text { Dentro } \\
\text { de sección }\end{array}$ & \\
\hline Diseño & Bajo & 5,3 & 2,4 & 5,0 & 4,1 \\
\hline \multirow[t]{2}{*}{ Organizacional } & Moderado & 76,3 & 95,2 & 95,0 & 89,2 \\
\hline & Alto & 18,4 & 2,4 & 0,0 & 6,7 \\
\hline Total & & 100,0 & 100,0 & 100,0 & 100,0 \\
\hline
\end{tabular}

Tabla 3. Niveles porcentuales del diseño organizacional por dimensiones.

\begin{tabular}{|c|c|c|c|c|c|}
\hline \multirow[b]{2}{*}{ Dimensiones } & \multirow[b]{2}{*}{ Niveles } & \multicolumn{3}{|c|}{ Secciones } & \multirow[b]{2}{*}{$\begin{array}{c}\text { Total } \\
\% \\
\text { Secciones }\end{array}$} \\
\hline & & $\begin{array}{l}\text { A } \\
\% \text { Dentro de } \\
\text { Sección }\end{array}$ & $\begin{array}{l}\text { B } \\
\% \text { Dentro de } \\
\text { sección }\end{array}$ & $\begin{array}{l}\mathrm{C} \\
\text { \% Dentro de } \\
\text { sección }\end{array}$ & \\
\hline \multirow{3}{*}{ Formalización } & Bajo & 2,6 & 16,7 & 12,5 & 10,8 \\
\hline & Moderado & 28,8 & 33,3 & 45,0 & 35,8 \\
\hline & Alto & 68,4 & 50,0 & 42,5 & 53,3 \\
\hline \multirow{3}{*}{ Profesionalismo } & Bajo & 0,0 & 4,8 & 2,5 & 2,5 \\
\hline & Moderado & 68,4 & 83,3 & 92,5 & 81,7 \\
\hline & Alto & 31,6 & 11,9 & 5,0 & 15,8 \\
\hline \multirow{3}{*}{ Tecnología } & Bajo & 65,8 & 71,4 & 37,5 & 58,3 \\
\hline & Moderado & 31,6 & 28,6 & 57,5 & 39,2 \\
\hline & Alto & 2,6 & 0,0 & 5,0 & 2,5 \\
\hline \multirow{3}{*}{$\begin{array}{l}\text { Entorno o } \\
\text { Medio } \\
\text { Ambiente }\end{array}$} & Bajo & 28,9 & 35,7 & 32,5 & 32,5 \\
\hline & Moderado & 50,0 & 59,5 & 60,0 & 56,7 \\
\hline & Alto & 21,1 & 4,8 & 7,5 & 10,8 \\
\hline \multirow{3}{*}{$\begin{array}{l}\text { Metas y } \\
\text { Estrategia }\end{array}$} & Bajo & 18,4 & 26,2 & 27,5 & 24,2 \\
\hline & Moderado & 47,4 & 61,9 & 70,0 & 60,0 \\
\hline & Alto & 34,2 & 11,9 & 2,5 & 15,8 \\
\hline \multirow{3}{*}{ Cultura } & Bajo & 10,6 & 4,8 & 5,0 & 6,7 \\
\hline & Moderado & 36,8 & 61,9 & 80,0 & 60,0 \\
\hline & Alto & 52,6 & 33,3 & 15,0 & 33,3 \\
\hline
\end{tabular}

Se observa, con respecto a las dimensiones formalización, profesionalismo y cultura, que el $89,1 \%, 97,5 \%$ y el $93,3 \%$, respectivamente, las encuestadas perciben un nivel moderado y alto en el diseño organizacional. Esto indica que dichas dimensiones conforme las percepciones, se vienen aplicando adecuadamente en la organización; es decir, se vienen aplicando las normas y reglamentos, manejando bien la documentación, existe un buen nivel de educación y capacitación, y el conjunto de valores, creencias, entendimientos y normas clave son compartidos por los colaboradores.
Por otro lado, existe un contraste con las dimensiones tecnología, entorno, $\mathrm{y}$, metas $\mathrm{y}$ estrategias. Se observa, que el $58,3 \%, 32,5 \%$, y el $24,2 \%$, respectivamente, las encuestadas perciben un nivel bajo en el diseño organizacional. Esto indica, que dichas dimensiones no se vienen aplicando adecuadamente en la organización o se aplican relativamente; es decir, existe un deficiente equipamiento en plataforma tecnológica, teniendo en consideración que con el avance científico tecnológico debe ser una prioridad en las organizaciones; las decisiones del entorno inciden negativamente en la formación policial, como es el 
incumplimiento en el tiempo de formación de las alumnas, que obviamente tiene sus repercusiones en el cumplimiento de las metas y objetivos como es la formación de calidad.

En la tabla 4, se hace una comparación de rangos promedio y estadístico de prueba Kruskal Wallis del diseño organizacional, donde se tienen la comparación entre los tres grupos de estudio (A, $\mathrm{B}$ y $\mathrm{C}$ ), que representan las secciones de la EPSB en cuanto al nivel de las percepciones del diseño organizacional. Se tiene al estadístico de prueba de Kruskal Wallis en concordancia a la prueba de independencia del Chi cuadrado de 7.418 y el valor de significación estadística de $\mathrm{p}=0.024$ menor al nivel de la significación estadística $\alpha=0.05(\mathrm{p}<\alpha)$ implicando que existen diferentes percepciones por secciones al nivel del diseño organizacional de la EPSB.

Tabla 4. Comparación de rangos promedio y estadístico de prueba Kruskal Wallis del diseño organizacional.

\begin{tabular}{llllll}
\hline & \multicolumn{6}{c}{ Rangos } \\
\hline & Sección & $\mathrm{N}$ & $\begin{array}{c}\text { Rango } \\
\text { promedio }\end{array}$ & Estadísticos de prueba ${ }^{\mathrm{a}, \mathrm{b}}$ \\
\hline \multirow{3}{*}{$\begin{array}{l}\text { Diseño } \\
\text { organizacional }\end{array}$} & sección A & 38 & 73,12 & Chi-cuadrado & 7,418 \\
\cline { 2 - 7 } & sección B & 42 & 53,54 & $\mathrm{gl}$ & 2 \\
\cline { 2 - 7 } & sección C & 40 & 55,83 & Sig. asintótica &, 024 \\
\cline { 2 - 7 } & Total & 120 & & & \\
\hline \multirow{2}{*}{$\begin{array}{l}\text { a Prueba de Kruskal Wallis. } \\
\text { bariable de agrupación: sección. }\end{array}$} & & & & \\
\end{tabular}

\section{Discusión}

El objetivo de esta investigación fue determinar las diferentes percepciones en el nivel del diseño organizacional, por secciones, en la EPSB. Los resultados del análisis realizado, mostraron que el diseño organizacional se encuentra en un nivel moderado y alto, y esto podría explicarse por la labor que viene realizando el director en la toma de decisiones en el cumplimiento de las metas y objetivos y que tienen repercusiones en las alumnas PNP en cuanto a sus percepciones. Coincidimos con Higuita et al. (2012) donde demuestran que algunas decisiones administrativas, como el diseño organizacional, necesarias para la eficiencia de la organización, tienen igualmente efectos en las personas, actualmente denominadas colaboradores. Estos resultados guardan similitud con el antecedente de Rodríguez et al. (2012) quienes plantean que a través del diseño organizacional se puede lograr mayor eficiencia y eficacia en las organizaciones, optimizando los recursos, tanto materiales como humanos. En ese orden de ideas, Bohle et al. (2018) hace mención la percepción de un alto nivel de apoyo organizacional intensifica el compromiso afectivo en la organización, creando más expectativas en los colaboradores.

En relación a los resultados en las dimensiones del diseño organizacional con niveles de moderado y alto en las dimensiones formalización, profesionalismo y cultura, se justifica en razón que la organización policial responde al modelo de paradigma burocrático que busca la eficiencia en la organización poniendo énfasis en las normas y los reglamentos, es decir la formalización, que están escritas en la organización, con una jerarquía de autoridad definida y que provienen según Tejada et el. (2020) de los estudios de Max Weber que propone un modelo de gestión organizacional basado precisamente en las normas y los reglamentos, con actividades bien definidas para lograr las metas y objetivos organizacionales. Azuero (2020) argumenta que este tipo de organización según Weber es semejante a una máquina, que se caracteriza por reglas, controles y jerarquías, y es promovida por la burocracia, en donde se plantea que el postulado que resume dicha teoría se refiere a que la mejor forma de organización es cuando esta tiene reglas claras y racionales, decisiones impersonales y empleados, y gestores con excelencia técnica, elementos que caracterizan la institución policial.

Con respecto al profesionalismo, coincidimos con Daft (2010) como el nivel de educación formal y capacitación de los empleados, donde las percepciones demuestran que la EPSB viene practicando en las alumnas y colaboradores una educación y capacitación constante, con condiciones de estudio aceptables, calidad en los docentes, encontrándose satisfechas profesionalmente en la escuela, como primer nivel del sistema educativo policial. Lorek et al. (2020), 
mostró que, es el nivel en donde la formación y el desarrollo de los colaboradores se debe gestionar adecuadamente, por tener una repercusión positiva en la seguridad social de un país.

La dimensión cultura, definida como el conjunto subyacente de valores, creencias, entendimientos y normas clave compartidos por los empleados (Daft, 2010), la EPSB propicia la participación, el consenso, compromiso en la institución policial y el espíritu de equipo, creando espacios para el desarrollo personal, social y cultural y valores que son compartidas por las alumnas, características que las diferencian de otras organizaciones y las hace más competitiva. Coincidimos con Robles et al. (2018) donde demuestran que estas particularidades son distintivas de cada organización y que constituyen una directriz para la gestión, el logro de objetivos y la forma de percibir el trabajo. De manera semejante Palomino et al. (2021) demuestran que existe una relación directa, positiva y significativa entre la cultura y el compromiso organizacional. En ese orden de ideas García et al. (2021) sostienen que es importante la cultura como un recurso para desarrollar sistemas innovadores para convertir en competitiva la organización. Los hallazgos en la dimensión cultura coinciden con Valle et al. (2017), donde se comprueba que la estructura y cultura organizacional influyen significativamente en los procesos de cambio.

Observando los resultados obtenidos en las dimensiones tecnología, entorno, y, metas y estrategias, se ha encontrado que estas se encuentran en un nivel bajo en el diseño organizacional. Conforme a las diferentes percepciones obtenidas, se puede analizar e interpretar que estas han permitido tener conocimiento respecto a los diferentes aspectos que forman parte de la formación que reciben las alumnas de la EPSB y que responden a la necesidad de contar con herramientas que permitan la integración de sistemas y tecnologías de información, y que se traducen en la falta de tecnificación del proceso de formación, con plataforma tecnológica acorde con el avance científico tecnológico que garantice desarrollar las actividades con estándares de calidad. Para corroborar este resultado, la investigación de Ferrer (2016) identificó que se deben incorporar elementos innovadores en la organización como factor clave para desarrollar procesos generadores de cambios, desarrollo de una estrategia exitosa y para adaptarse a los cambios que se generan en el ambiente como consecuencia del desarrollo económico, social y tecnológico, a nivel mundial, que se ven reflejados en el diseño organizacional. Asimismo, los hallazgos son coherentes con la investigación de Villalobos (2020) donde estableció que los procesos de innovación tecnológica permiten desarrollar y fortalecer las competencias profesionales y habilidades en la solución de los diferentes problemas en el servicio policial, a partir de la etapa de formación policial, hacia una fuerza pública más eficiente y que tengan un fuerte impacto en los índices delictivos.

Con respecto a las percepciones obtenidas en relación al entorno, se puede analizar e interpretar que las disposiciones del Comando policial, como son el nombramiento de las comisiones y los servicios externos a la escuela, periodo de formación, influyen negativamente en el cumplimiento de los objetivos como es la formación policial de calidad; por lo tanto, la organización debe adaptarse a las disposiciones existentes en las normas y que vienen del entorno, establecidas en los escalones superiores. Hoy más que nunca los administradores se ven sujetos a una presión cada vez mayor para prever y responder a este conjunto de fuerzas externas, que favorecen o entorpecen con el accionar de sus fuerzas, el desarrollo de la actividad empresarial (Castro, 2007).

Por último, conforme a las diferentes percepciones obtenidas con relación al diseño organizacional en la dimensión metas y estrategias, que son establecidas en la alta dirección de la EPSB, teniendo en consideración las fuerzas endógenas y exógenas, se puede analizar e interpretar que no se conoce la misión, la visión, los objetivos, así como el plan de acción que orienten el accionar de los colaboradores como son las alumnas PNP y puedan definir el propósito de la escuela y las técnicas competitivas que la diferencien de otras organizaciones. El diseño de las organizaciones en el contexto actual tiene que apoyar los requerimientos de la estrategia, ser consistentes con las demandas del entorno, para garantizar la funcionalidad organizacional (Castellanos y Castellanos, 2011). En este contexto, el conocimiento de todos los procedimientos al interior de la organización pasa por el ejercicio de un buen liderazgo, que conforme Rivera et al. (2018) el éxito de las mejores compañías del mundo, se debe al liderazgo que se ejerce en la organización. 


\section{Conclusiones}

Existen diferentes percepciones por secciones al nivel del diseño organizacional de la Escuela de Policía San Bartolo, conforme lo demuestra la prueba Kruskal Wallis, que de acuerdo con los resultados obtenidos, el diseño organizacional se encuentran en un nivel moderado y alto, niveles que se ven corroborado en las dimensiones: formalización, profesionalismo y cultura, que es una práctica frecuente en la Policía Nacional del Perú, donde la gestión está orientada al cumplimiento de la legalidad y esto es determinado por los documentos que se generan para lograr orden y eficiencia en la organización; las alumnas manifiestan la educación y capacitación que reciben así como la práctica de valores como la disciplina, responsabilidad, entendimientos, normas clave compartidas como parte de la cultura organizacional.

Respecto a las percepciones de las dimensiones: tecnología, entorno y, metas y estrategias, en el diseño organizacional, estas se encuentran en un nivel bajo, evidencian deficiencias en plataforma tecnológica y que las decisiones del entorno que se traducen en disposiciones para la reducción del tiempo de formación policial, comisiones, repercuten en el cumplimiento de metas y objetivos organizacionales como es la formación de calidad y por ende la prestación un buen servicio a la comunidad.

\section{Referencias bibliográficas}

Azuero, A. R. (2020). Evaluación epistemológica a la teoría de la burocracia de Max Weber. Revista Espacios, 41(45), 338-353. 10.48082/ espacios-a20v41n $45 \mathrm{p} 27$

C. B. Barona and M. R. Ramirez, "Design and Development in Advanced Manufacturing processes from an Organizational Innovation perspective," 2020 15th Iberian Conference on Information Systems and Technologies (CISTI), 2020, pp. 1-6, doi: 10.23919/ CISTI49556.2020.9140959.

Barrera, J. (2016). Police studies in Latin America. Some theoretical and methodological limitations in their approach a direct target. Revista Criminalidad, 58(3), 119-132.
Besri Z., Boulmakoul A. (2021) An Intuitionist Fuzzy Method for Discovering Organizational Structures that Support Digital Transformation. In: Kahraman C., Cevik Onar S., Oztaysi B., Sari I., Cebi S., Tolga A. (eds) Intelligent and Fuzzy Techniques: Smart and Innovative Solutions. INFUS 2020. Advances in Intelligent Systems and Computing, 1197. Springer, Cham. 10.1007/978-3-030-51156-2 39

Bohle, S. A., Chambel, M., J., Medina, F. M. \& Da Cumba, B. S. (2018). Papel da percepção do suporte organizacional na insegurança e desempenho no trabalho. Revista de Administracao de Empresas, 58(4), 393-404. $\underline{10.1590 / \mathrm{S} 0034-759020180405}$

Bond, B. (2020). Organizational Change in an Urban Police Department: Innovating to Reform. Organizational Change in an Urban Police Department: Innovating to Reform $\left(1^{\text {st }}\right.$ ed.). Routledge. 10.4324/9781315641225

Buderath, M., Heath, M. (2021). Fuerza Civil: Capital Accumulation and Social Control in Nuevo León, Mexico. Latin American Perspectives, 48(1), 163-183. $\underline{10.1177 \% 2 \mathrm{~F} 0094582 \mathrm{X} 20975011}$

Castellanos, J. R. y Castellanos C. A. (2011). El diseño organizacional: evolución y perspectivas. Revista Ciencia y Técnica Administrativa, 10(2), 1666-1680.

Castro, Y. (2007). La influencia del entorno en el desempeño de las organizaciones. Particularidades del entorno empresarial cubano. Economía y Desarrollo, 142(2), 139149.

Castro, M. T. (2020). Organizational design through a systemic and cybernetic approach: The case of an enterprise of sanitation. Ingenlare, 28(1), 68-82. 10.4067/S0718$\underline{33052020000100068}$

Cornejo, V. \& Flores, S. (2020). Model for organizational design based on qualities (Modelo del diseño organizacional basado en cualidades. Revista Ibérica de sistemas e tecnologías de informacao, 2020(27), 733750 . 
Chiavenato, I. (2006). Introducción a la Teoría General de Administración. 7ma ed. México: McGraw- Hill/Interamericana Editores, S.A. de C. V.

Dammert, L. y Castañeda, M. (2019). ¿Marketing o efectividad? Policía comunitaria en Perú. Utopía y Praxis Latinoamericana, 24(2), 5167. 10.5281/zenodo.3344846

Daft, R.L. (2010). Teoría y diseño organizacional. 11 ava ed. México: Cengage Learning.

Díaz, J. R., Núñez, L. A. y Cáceres, K. V. (2018). Influencia de las competencias gerenciales y la gestión por resultados en la imagen institucional. Difusión cultural y científica de la Universidad La Salle en Bolivia, 16(16), 169-197.

Estupiñan, J., Martinez, A., Acosta, R., Villacres, A., Escobar, J. \& Batista, N. (2018). Management system of higher education in ecuador. impact on the learning process. Dilemas Contemporáneos: Educación, Política y Valore, Tomo VI, art. 24.

Ferrer, M.A. (2016). Innovation and organizational design. Innovación y diseño organizacional. Revista Venezolana de Gerencia, 21(74), 190192.

García, J., Tumbajulca, I., \& Cruz, J. (2021). Innovación organizacional como factor de competitividad empresarial en mypes durante el Covid-19. Comuni@cción: Revista De Investigación en Comunicación y Desarrollo, 12(2), 99-110. 10.33595/2226-1478.12.2.500

Gareth, J. (2008). Teoría Organizacional, diseño y cambio en las organizaciones. 5 ta ed. México: Pearson Educación.

Hernández, R., Fernández C., y Baptista (2014). Metodología de la investigación. 6ta ed. México: McGraw-Hill/Interamericana Editores, S. A. de C. V.

Higuita, D. (2011). Parámetros de diseño organizacional: incidencia en la productividad y en los vínculos sociales. Revista Facultad de Ciencias Económicas: Investigación y reflexión, 19(1), 73-90.
Lorek, M., Piecuch, T., Itrich, J., \& Minkina, M. (2020). Management of the police officers training system and the effectiveness of internal security. Journal of Security and Sustainability Issues, 9(4), 1337-1348. 10.9770/jssi.2020.9.4(19)

Pacheco-Ruíz, Carlos, Rojas-Martínez, Claudia, Niebles-Nuñez, William, \& HernándezPalma, Hugo G. (2020). Técnicas de desarrollo organizacional para la actualización tecnológica del docente en instituciones de educación superior. Formación universitaria, 13(5), 191-202. 10.4067/S0718$\underline{50062020000500191}$

Palomeque, I. \& Ruiz, J. (2019). Impact of institutional management on the generation of scientific knowledge in higher education institutions. Espacios, 40(2), 14p. https:// www.revistaespacios.com/a19v40n02/ a19v40n02p14.pdf

Palomino, A., Velásquez, V., Quintana, F. \& Ambrosio, A. (2021). Organizational culture and commitment: Indirect effects of the employer brand experience. Universidad y Sociedad, 13(4), 369-377.

Quintero, S. P. (2020). Citizen security and community participation in Latin America. RevistaCientífica GeneralJoséMaríaCórdova, 18(29), 5-21. 10.21830/19006586.561 http:// www.scielo.org.co/scielo.php?pid=S1900-

Quispe, A., Calla, K. M., Yangali, J. S., Rodriguez, J. L. y Pucamayo, I. I. (2019). Estadística no paramétrica aplicada a la investigación cientifica. 1ra ed. Colombia: EIDEC.

Rivera, D. A., Berbesi, Y. M. \& Ramirez, M. L. (2018). Georeferencing and its influence in organizational leadership: A look from the development of managerial skills. Espacios, 39 (34).

Robles, C., Montes, J., Rodríguez, A. \& Ortega, A. (2018). Diseño y validación de un instrumento de cultura organizacional para empresas medianas. Nova Scientia, 10(21), 552-575. $\underline{10.21640 / \text { ns.v10i21.1453 }}$ 
Rodríguez, I., González, A., Noy, P., \& Pérez, S. (2012). Metodología de Diseño Organizacional integrando enfoque a procesos y competencias. Ingeniería Industrial, 33(2), 188-199.

Serkan, N., Kanan, S., Bayraktar, Özgü, Emiroğlu, O., \& Güneyli, A. (2019). Levels of readiness in total quality management. Amazonia Investiga, 7(16), 268-275.

Stoner, J., Freeman, R. y Gilbert, D. (1999). Administración. 6ta Ed. México: Prentice Hall Hispanoamericana, S.A.

Tejada, G., Rengifo, R., Boy, A. \& Rodríguez, J. (2020). Organizational structure and control in a bureaucratic management model. Revista Venezolana de Gerencia, 25(89), 66-78. $\underline{10.37960 / \text { revista.v25i89.31385 }}$

Ugarte, M., Rivero, J. y Bautista, D. (2021). ¿Por qué las reformas administrativas no logran los resultados esperados? https:// escuela.pucp.edu.pe/gobierno/wp-content/ uploads $/ 2021 / 06 / 2$.dp administracion publica.pdf
Valderrama, S. (2013). Pasos para elaborar proyectos de investigación cientifica. $2 \mathrm{da}$ ed. Perú: San Marcos E. I. R. L., editor.

Valle, A. T., Proaño, T. D. y Cruz, M. E. (2017). Estructura, Cultura y Cambio Organizacional Cultura - Cambio - Forma - Fuerza. Revista Cientifica Hermes, 18,304-324.

Vera, A. y Blanco, A. B. (2019). Model for human talen manegement in service-sector smes in Barranquilla, Colombia. Innovar, 29(74), 2555. 10.15446/innovar.v29n74.82059

Villalobos, H. (2020). Technological Development in Police Matters: A Successful Recipe for Crime Prevention. Revista de Relaciones Internacionales, Estrategia y Seguridad, 15(1), 79-97. 10.18359/ries.4243

Willis, J. J., Koper, C. S. \& Lum, C. (2020) Technology use and constituting structures: accounting for the consequences of information technology on police organisational change. Policing and Society, 30(5), 483-501. $\underline{10.1080 / 10439463.2018 .1557660}$ 